\title{
Direct observations of nitric oxide produced by energetic electron precipitation into the Antarctic middle atmosphere
}

\author{
David A. Newnham, ${ }^{1}$ Patrick J. Espy, ${ }^{2}$ Mark A. Clilverd, ${ }^{1}$ Craig J. Rodger, ${ }^{3}$ \\ Annika Seppälä, ${ }^{1,4}$ David J. Maxfield, ${ }^{1}$ Paul Hartogh, ${ }^{5}$ Kim Holmén, ${ }^{6}$ \\ and Richard B. Horne ${ }^{1}$ \\ Received 7 July 2011; revised 19 September 2011; accepted 21 September 2011; published 19 October 2011.
}

[1] We report the first ground-based passive microwave observations made from Troll station, Antarctica, which show enhanced mesospheric nitric oxide (NO) volume mixing ratio reaching levels of 1.2 ppmv, or $2-3$ orders of magnitude above background, at $70-80 \mathrm{~km}$ during small, relatively isolated geomagnetic storms in 2008. The mesospheric NO peaked 2 days after enhanced NO at higher altitudes $(110-150 \mathrm{~km})$ measured by the SABER satellite, and 2 days after peaks in the $>30 \mathrm{keV}$ and $>300 \mathrm{keV}$ electron flux measured by POES, although the $300 \mathrm{keV}$ electron flux remained high. High time resolution data shows that mesospheric NO was enhanced at night and decayed during the day and built up to high levels over a period of 3-4 days. The altitude profile of mesospheric NO suggests direct production by $\sim 300 \mathrm{keV}$ electron precipitation. Simulations using the Sodankylä Ion and Neutral Chemistry model show that the delay between thermospheric and mesospheric NO enhancements was primarily a result of the weaker production rate at lower altitudes by $\sim 300 \mathrm{keV}$ electrons competing against strong day-time losses. Citation: Newnham, D. A., P. J. Espy, M. A. Clilverd, C. J. Rodger, A. Seppälä, D. J. Maxfield, P. Hartogh, K. Holmén, and R. B. Horne (2011), Direct observations of nitric oxide produced by energetic electron precipitation into the Antarctic middle atmosphere, Geophys. Res. Lett., 38, L20104, doi:10.1029/2011GL048666.

\section{Introduction}

[2] The odd nitrogen $\left(\mathrm{NO}_{\mathrm{x}}\right)$ species nitric oxide $(\mathrm{NO})$ and nitrogen dioxide $\left(\mathrm{NO}_{2}\right)$ are produced in the middle atmosphere by precipitating energetic electrons and protons [Brasseur and Solomon, 2005]. In the thermosphere and upper mesosphere $\mathrm{NO}_{\mathrm{x}}$ exists mainly as $\mathrm{NO}$ but below $70 \mathrm{~km}$ conversion to $\mathrm{NO}_{2}$ occurs [Solomon et al., 1982]. The chemical lifetime of NO in the sunlit mesosphere and lower thermosphere is typically one day [Solomon et al., 1999] although between $55 \mathrm{~km}$ and $85 \mathrm{~km}$ the lifetime may be as short as one hour [Shimazaki, 1984]. In darkness $\mathrm{NO}_{\mathrm{x}}$ is

\footnotetext{
${ }^{1}$ British Antarctic Survey, Cambridge, UK.

${ }^{2}$ Department of Physics, Norwegian University of Science and Technology, Trondheim, Norway.

${ }^{3}$ Department of Physics, University of Otago, Dunedin, New Zealand.

${ }^{4}$ Also at Earth Observation Unit, Finnish Meteorological Institute, Helsinki, Finland.

${ }^{5}$ Max Planck Institute for Solar System Research, KatlenburgLindau, Germany.

${ }^{6}$ Norwegian Polar Institute, Tromsø, Norway.
}

Copyright 2011 by the American Geophysical Union. 0094-8276/11/2011GL048666 much longer lived and can be transported downward by the polar vortex at high latitudes during winter [Siskind et al., 2000]. Atmospheric circulation models and re-analysis meteorological data have indicated that changes in ozone abundance due to $\mathrm{NO}_{\mathrm{x}}$ arising from energetic particle precipitation can affect polar surface temperatures by as much as 4 K [Rozanov et al., 2005; Seppälä et al., 2009].

[3] $\mathrm{NO}_{\mathrm{x}}$ may be produced more frequently and persistently by energetic electron precipitation (EEP) from the Earth's magnetosphere than by solar protons [Randall et al., 2005]. However, it is unclear which electron energy range is most important for stratospheric chemistry. At auroral geomagnetic latitudes $\left(70^{\circ}<\Lambda<75^{\circ}\right)$ high flux of low energy (1-10 keV) electrons enter the atmosphere almost continuously [Baker et al., 2001] and are enhanced during substorms at night. However meridional circulation in the upper mesosphere and lower thermosphere has been observed to show large variability and equatorward flow above $80-98 \mathrm{~km}$ at $68^{\circ} \mathrm{S}$ in winter [Sandford et al., 2010], which could act as a barrier to auroral NO entering the polar vortex and being efficiently transported downwards.

[4] Higher-energy (10 keV-several $\mathrm{MeV})$ electrons precipitate from the radiation belts in the subauroral zone $\left(\Lambda \leq 75^{\circ}\right)$ during magnetic storms, particularly in the southern hemisphere and pole-ward of the South Atlantic Anomaly (SAA) [Horne et al., 2009]. Although in general the precipitating flux decreases rapidly with increasing electron energy this mechanism can produce $\mathrm{NO}_{\mathrm{x}}$ directly in the stratosphere and mesosphere [Turunen et al., 2009].

[5] While $\mathrm{NO}_{\mathrm{x}}$ has been observed using satellite instruments such as the microwave limb sounder Odin-SMR [Murtagh et al., 2002], here we present vertical profiles of NO measured directly with unprecedented temporal resolution by a new passive microwave radiometer located poleward of the SAA, in Antarctica. The high time and altitude resolution enable us to determine the electron energy responsible for $\mathrm{NO}_{\mathrm{x}}$ production.

\section{Microwave Radiometer Experimental Setup}

[6] The microwave radiometer used in this study has been described previously [Espy et al., 2006] and only the NO measurement details are given here. Ground-based atmospheric observations at a $60^{\circ}$ zenith angle were made from Troll station, Antarctica $\left(72^{\circ} 01^{\prime} \mathrm{S}, 02^{\circ} 32^{\prime} \mathrm{E}, 1275 \mathrm{~m}\right.$ above sea level). Troll is at a geomagnetic latitude of $65^{\circ}$, suitable for observing the effects of EEP from the outer radiation belt, and it is also typically inside the Antarctic polar vortex which extends to $60^{\circ} \mathrm{S}$ and from the mesosphere to approximately $16 \mathrm{~km}$ [Turunen et al., 2009]. 

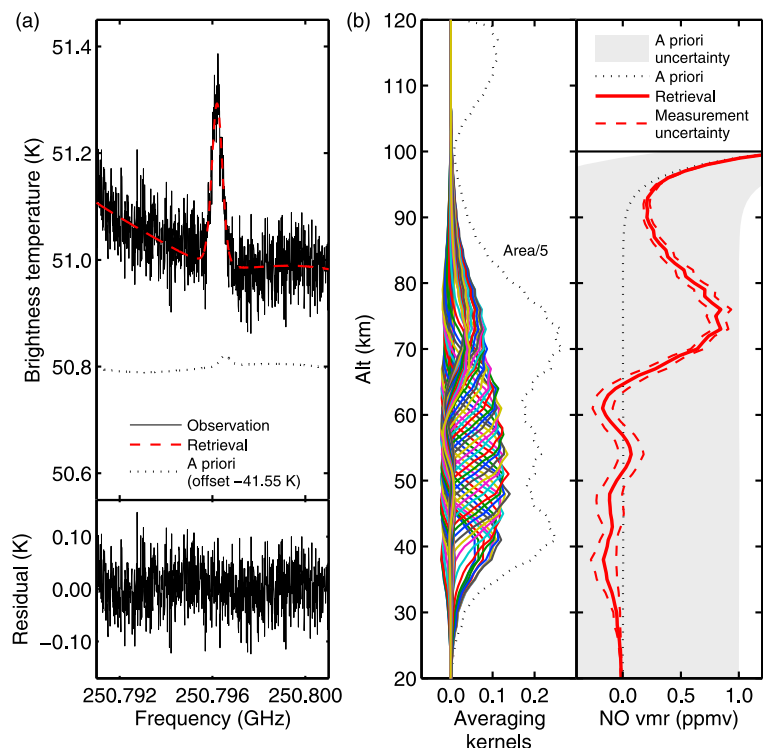

Figure 1. (a) Example microwave brightness temperature spectrum for 2008 day 89, together with initial forward model (a priori), retrieved, and residual spectra and (b) averaging kernels for the inversion and a priori and retrieved NO profiles corresponding to the data in Figure 1a, both at $1 \mathrm{~km}$ intervals.

[7] NO volume mixing ratio (VMR) profiles were inverted from calibrated, brightness temperature spectra of $14 \mathrm{kHz}$ resolution and $10 \mathrm{MHz}$ width, centered on the NO line at 250.796 GHz, using the Microwave Observation Line Estimation and Retrieval (MOLIERE) version 5 code [Urban et al., 2004]. A priori pressure, temperature, ozone, water vapor, and NO profiles above $30 \mathrm{~km}$ were calculated using the Sodankylä Ion and Neutral Chemistry (SIC, version 6.8) model [Verronen et al., 2002] under geomagneticallyquiet conditions. For altitudes up to $30 \mathrm{~km}$ MIPAS/Envisat (Michelson Interferometer for Passive Atmospheric Sounding) data were combined with 10-year (1999-2008) averages of ozonesonde data from Neumayer station $\left(70^{\circ} 39^{\prime} \mathrm{S}, 08^{\circ} 15^{\prime} \mathrm{W}\right)$. Spectroscopic reference data for radiative transfer calculations were taken from HITRAN 2008 [Rothman et al., 2009]. Data inversion was performed from the ground to $120 \mathrm{~km}$ with $1 \mathrm{~km}$-thick layers to ensure stable convergence. NO and water vapor profiles were adjusted in the forward model calculations to provide the best fit to the observed brightness temperatures. Radiometer data affected by weather conditions, or negative VMR values, were rejected.

[8] Figure 1a shows the atmospheric emission averaged for 2008 day 89 (29 March) where the maximum brightness-temperature change at $250.796 \mathrm{GHz}$ due to NO is $0.3 \mathrm{~K}$. The residual brightness temperature, i.e. the difference between the observation and final forward model spectrum using the retrieved parameters, shown in Figure 1a (bottom) indicates that the observational data are fitted to within the measurement uncertainty due to baseline noise of $0.03 \mathrm{~K}$. The area of the normalized averaging kernels (Figure 1b) [Rodgers, 2000] is $\geq 0.5$ for atmospheric layers between $35-83 \mathrm{~km}$, indicating good information retrieval, although the measurements contribute to the retrieved NO VMR up to at least $90 \mathrm{~km}$. The vertical resolution is estimated from the width of the averaging kernels to be $8 \mathrm{~km}$.
The retrieved NO VMRs shown in Figure $1 \mathrm{~b}$ (right) are much higher than the a priori values for $70-90 \mathrm{~km}$ and reach $0.8 \mathrm{ppmv}$ at $75-79 \mathrm{~km}$. The measurement uncertainties shown in Figure 1b (right) are the diagonal elements of the measurement error covariance matrix. The NO VMR measurement error at each $1 \mathrm{~km}$ altitude level between $60 \mathrm{~km}$ and $90 \mathrm{~km}$ is $\pm 0.1 \mathrm{ppmv}$ for daily averages and $\pm 0.3 \mathrm{ppmv}$ for 3-hour averages. Instrument calibration and smoothing errors are not included in the current analysis and will be the subject of future work. Uncertainties in the pressure profile, or the air-broadening coefficient of the NO line, lead to a $3 \mathrm{~km}$ uncertainty in the NO altitude profiling. A $10 \%$ uncertainty in the atmospheric temperature profile changes the retrieved NO abundances by $30 \%$, which is $1-2$ orders of magnitude smaller than the NO variations shown later in the paper.

\section{NO Observations}

[9] The ground-based microwave observations for 2008, days 80-129 (20 March to 8 May) are compared in Figure 2 with NO $5.3 \mu \mathrm{m}$ volume emission rate (VER) data for the lower thermosphere $(100-150 \mathrm{~km})$ from the SABER satellite instrument [Mlynczak, 1997]. Periods of enhanced NO VER can be seen (Figure 2a) at altitudes above $110 \mathrm{~km}$ where significant non-LTE $5.3 \mu \mathrm{m}$ radiance occurs and can be measured by SABER. This occurs most strongly between days 86-90 and 114-118, but also less strongly on days 96-101 and 107-108. These coincide with increases in geomagnetic activity index Ap (Figure 2b). The two periods of highest VER occur within 24 hours of the days (87-88 and 114) with highest Ap and exhibit the 27-day repeatability that is associated with recurrent geomagnetic activity [Borovsky and Denton, 2006]. Higher thermospheric NO VER also corresponds closely with increased daily average electron count rate for the $>30 \mathrm{keV}$ channel of the SEM-2 MEPED instrument onboard the low altitude $(\sim 800 \mathrm{~km})$ POES satellites at $60^{\circ}<\Lambda<65^{\circ}$ (Figure 2b).

[10] The radiometer observations (Figure 2c) also show periods of enhanced NO, lasting 3-5 days, but at lower altitudes in the range $70-85 \mathrm{~km}$. Daily average VMR reaches approximately $1.2 \mathrm{ppmv}$ at $73-80 \mathrm{~km}$ on day 91 , approximately two to three orders of magnitude above typical background levels (10-100 ppbv) at this time of year. A particularly striking feature is that the enhanced NO VMR below $85 \mathrm{~km}$ occurs 2-3 days after the enhancement above $110 \mathrm{~km}$.

[11] The 70-85 km altitude where NO VMR is enhanced (Figure 2c) corresponds to a peak in ionization produced by $\sim 300 \mathrm{keV}$ electrons [Turunen et al., 2009], assuming precipitation by a mono-energetic electron beam. However, the MEPED $>300 \mathrm{keV}$ electron count rate (Figure $2 \mathrm{~d}$ ) reaches a maximum 1-2 days before the peak in VMR at $70-80 \mathrm{~km}$ and then decreases after day 88 while NO continues to increase. Although we make use of MEPED measurements of the $90^{\circ}$ telescope "trapped" electrons (and not the $0^{\circ}$ "precipitating" measurements due to strong contamination in this telescope [Rodger et al., 2010]), our experience shows that the precipitation generally follows variations in the trapped electron flux.

[12] Correlation coefficient values were calculated by comparing the 50-day observed NO data-sets with the Ap index and MEPED electron count rate data time shifted by a 


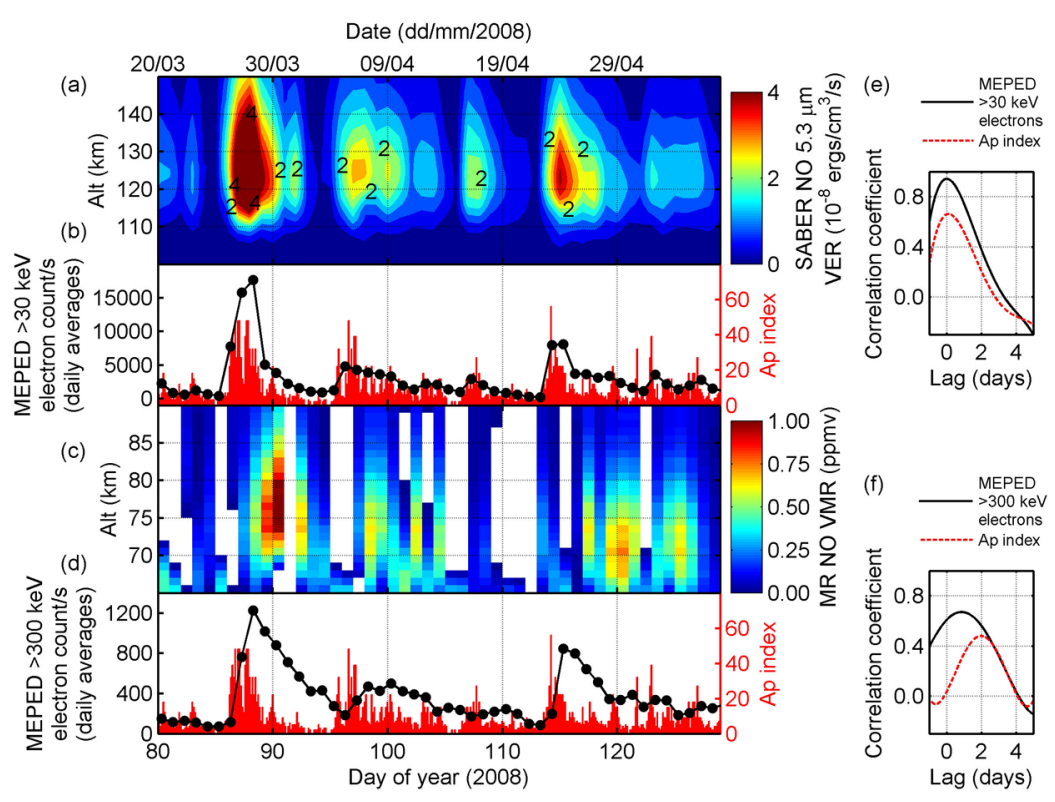

Figure 2. (a) SABER NO $5.3 \mu \mathrm{m}$ VER for $65^{\circ} \mathrm{S}-75^{\circ} \mathrm{S}$, (b) MEPED $>30 \mathrm{keV}$ channel trapped and quasi-trapped electron flux and 3-hour average Ap index, (c) Microwave radiometer daily average NO VMR, (d) MEPED $>300 \mathrm{keV}$ channel trapped and quasi-trapped electron flux and 3-hour average Ap index, (e) Correlation between the SABER NO $5.3 \mu \mathrm{m}$ VER for $65^{\circ} \mathrm{S}-75^{\circ} \mathrm{S}$ averaged over $120-130 \mathrm{~km}$ and the time-shifted Ap index and MEPED $>30 \mathrm{keV}$ channel trapped and quasi-trapped electron flux time series, and (f) Correlation between the microwave radiometer daily NO VMR averaged over 70-80 km and the time-shifted Ap index and MEPED $>300 \mathrm{keV}$ channel trapped and quasi-trapped electron flux time series.

range of lag times. The largest correlation, with $r=0.94$, was calculated with a lag time of 0.0 days between the NO SABER VER and $>30 \mathrm{keV}$ electron count rate, as shown in Figure 2e. For SABER NO VER and Ap index the largest correlation is $r=0.66$ for a lag time of 0.1 days. The radiometer NO VMR data is moderately correlated with
$>300 \mathrm{keV}$ electron count rate $(r=0.67$, lag time of 0.9 days $)$ and Ap index ( $r=0.48$, lag time of 2.0 days), as seen in Figure $2 f$.

[13] To understand NO variability in more detail, Figure $3 b$ shows higher resolution, 3-hourly profiles for the most intense geomagnetic storm period, days 86-94 (26 March to

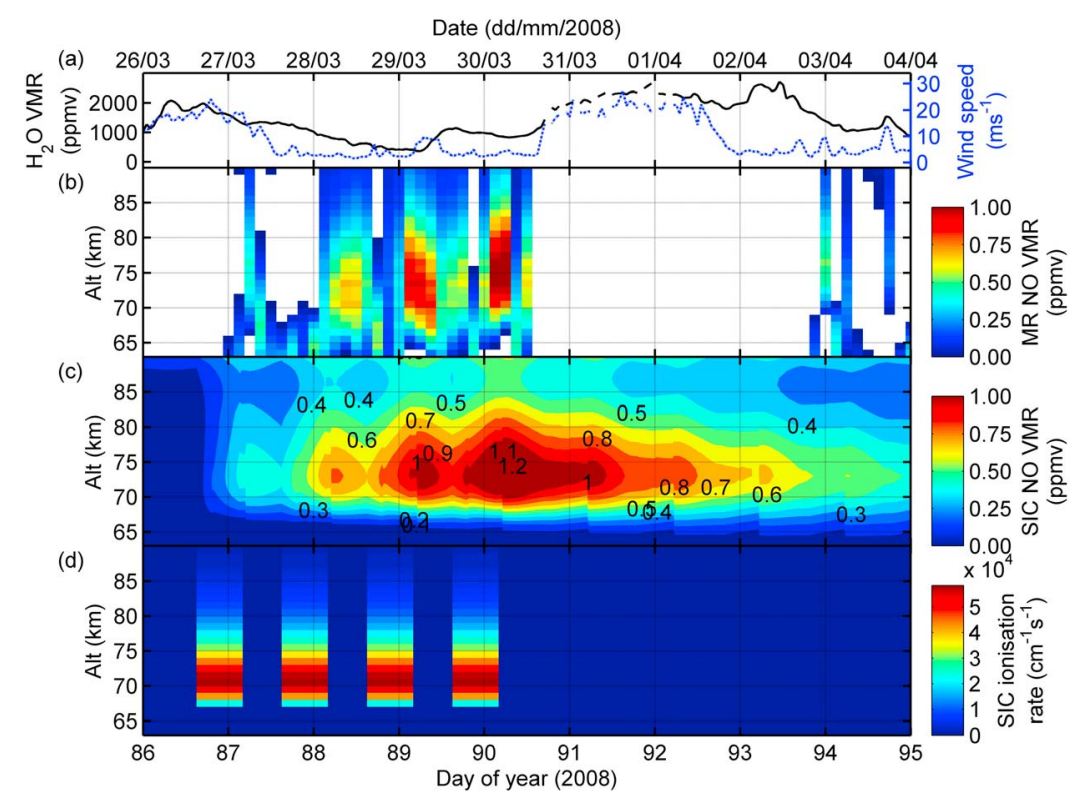

Figure 3. (a) Surface-level water vapour VMR and wind speed from meteorological observations at Troll station, Antarctica, where higher values lead to attenuation of the NO signal received by the microwave radiometer; (b) microwave radiometer 3-hour average NO VMR; (c) SIC model NO VMR; and (d) ionization rate profile used in SIC model to generate result shown in Figure 3c. 
4 April 2008). NO increases during day 88, diminishes during sunlit hours and continues to increase each night until day 90 . Thereafter the microwave signal was attenuated by increased surface-level humidity (days $90.5-94$ ) and possibly by blown snow when wind speeds were above $20 \mathrm{~ms}^{-1}$ (Figure 3a). The $\mathrm{H}_{2} \mathrm{O}$ profile was adjusted in the radiative transfer model to reproduce the observed attenuation during this period but this meant that limited NO information could be retrieved from the 3-hour averages under these conditions. The daily profiles (Figure 2c) are more robust, due to the longer integration time, and confirm that NO remained above 0.5 ppmv between days 90.5 and 93.0 with recovery to background levels by the end of day 94 .

\section{Ion-Neutral Chemistry Modeling}

[14] Two key processes that could account for the observed diurnal variability of NO in the upper mesosphere are production by EEP and loss by photolysis [Brasseur and Solomon, 2005]. To test this the SIC model [Verronen et al., 2002] was used to calculate NO VMR for different flux of $300 \mathrm{keV}$ electrons. There is currently large uncertainty in how the flux of different electron energies varies with magnetic local time (MLT). During large geomagnetic storms $>150 \mathrm{keV}$ electron precipitation fluxes have been observed to be higher on the dayside than at night [Rodger et al., 2007], whereas studies combining averages of satellite data and model data show $30 \mathrm{keV}-1 \mathrm{MeV}$ precipitation tending to maximize near dawn [Lam et al., 2010]. However, statistical analysis of direct POES observations shows $>300 \mathrm{keV}$ precipitation remaining high throughout the night during the main phase of geomagnetic storms [Horne et al., 2009]. Therefore we applied precipitation in the SIC model for 4 consecutive nights between 1500-0300 UTC, corresponding to days 87-90 when the MEPED $>300 \mathrm{keV}$ electron count rate was $>750 \mathrm{~s}^{-1}$. The model was run with different $300 \mathrm{keV}$ electron flux levels and timings but only the results for a total precipitation flux of $3.14 \times 10^{6} \mathrm{~cm}^{-2} \mathrm{~s}^{-1}$ are shown. Runs with lower electron flux yielded peak NO VMR over the same altitude range, i.e. $70 \mathrm{~km}$ to $80 \mathrm{~km}$, but with less accumulation over consecutive days due to the linear response of NO production to ionization rate. Reducing the duration of the night-time precipitation pulses also led to greater diurnal variability of NO.

[15] Figure 3c shows that the NO calculated by the SIC model has a diurnal variation that is similar to the microwave observations and which gradually increases at $70-80 \mathrm{~km}$ over a period of 3-4 days. However, the model VMR does not decay in sunlight to the low levels measured by the radiometer. On days $88-90$ the model NO VMR does not drop below $0.6 \mathrm{ppmv}$, somewhat above the estimated measurement detection limit of $0.3 \mathrm{ppmv}$. This suggests that either the loss in sunlight is underestimated, which is unlikely, or that electron precipitation in the model should be lower, or have a much larger diurnal variation. Higher resolution data are required to test this and are not yet available. When the EEP is switched off in the model after day 90 the NO decays back to near-background levels by the end of day 94 , as observed by the radiometer. The NO above $87 \mathrm{~km}$ is due to solar soft X-rays $(2-10 \mathrm{~nm})$ [e.g. Solomon et al., 1982] and much weaker ionization produced by the $300 \mathrm{keV}$ electrons. Model runs with and without the energetic electron source, while keeping all other forcings unchanged, confirm that $\sim 300 \mathrm{keV}$ electrons, rather than solar soft X-rays, are the main source of NO production below $87 \mathrm{~km}$.

\section{Conclusion}

[16] The data show that while NO above $110 \mathrm{~km}$ can be directly related to enhanced $>30 \mathrm{keV}$ electron flux, increases in mesospheric $(70-80 \mathrm{~km}) \mathrm{NO}$ was delayed by 1-2 days with respect to the enhancements in the $>30 \mathrm{keV}$ and $>300 \mathrm{keV}$ electron flux. However, higher (3-hour) resolution data and modeling strongly suggest that between 2008 , days 86 and 94 (26 March to 4 April) the NO at 70-80 km was produced directly by the precipitation of $\sim 300 \mathrm{keV}$ electrons. The diurnal variation and gradual build up of mesospheric NO over 3-4 days, rather than an immediate increase with electron flux, indicate that production was competing strongly against day-time losses. Thus the delay between thermospheric and mesospheric NO enhancements was primarily a result of the weaker production rate at lower altitudes by $\sim 300 \mathrm{keV}$ electrons. More detailed calculations could be carried out using daily measured $>300 \mathrm{keV}$ electron flux as the time dependent driver for the model, although comparison with observations may be limited by the lack of 3-hour average NO VMR data after day 90, as well as the \pm 0.3 ppmv uncertainty and detection limit of these measurements.

[17] The data and simulations did not show evidence of downward descent from higher altitudes, so that even weak geomagnetic storms can directly elevate mesospheric NO abundance to $1.2 \mathrm{ppmv}$, which is two to three orders of magnitude above the background level at this time of year. Were such $\mathrm{NO}_{\mathrm{x}}$ production by EEP to occur during wintertime, significant $\mathrm{NO}$ and $\mathrm{NO}_{2}$ could accumulate in the highlatitude mesosphere and be transported vertically downwards within the polar vortex to the stratosphere.

[18] Acknowledgments. AS was supported by the European Commission project FP7-PEOPLE-IEF-2008/237461. We thank Atle Markussen, Paul Breen, and Joachim Urban for their help. The Norwegian Institute for Air Research (NILU) and The Research Council of Norway are acknowledged for providing meteorological data. This work was supported in part by the UK Natural Environment Research Council. The authors thank the anonymous referees for their valuable comments.

[19] The Editor wishes to thank two anonymous reviewers for their assistance evaluating this paper.

\section{References}

Baker, D. N., C. A. Barth, K. E. Mankoff, S. G. Kanekal, S. M. Bailey, G. M. Mason, and J. E. Mazur (2001), Relationships between precipitating auroral zone electrons and lower thermospheric nitric oxide densities: 1998-2000, J. Geophys. Res., 106, 24,465-24,480, doi:10.1029/ 2001JA000078.

Borovsky, J. E., and M. H. Denton (2006), Differences between CMEdriven storms and CIR-driven storms, J. Geophys. Res., 111, A07S08, doi:10.1029/2005JA011447.

Brasseur, G. P., and S. Solomon (2005), Aeronomy of the Middle Atmosphere, 3rd ed., Springer, Dordrecht, Netherlands.

Espy, P. J., P. Hartogh, and K. Holmen (2006), A microwave radiometer for the remote sensing of nitric oxide and ozone in the middle atmosphere, Proc. SPIE, 6362, 63620P, doi:10.1117/12.688953.

Horne, R. B., M. M. Lam, and J. C. Green (2009), Energetic electron precipitation from the outer radiation belt during geomagnetic storms, Geophys. Res. Lett., 36, L19104, doi:10.1029/2009GL040236.

Lam, M. M., R. B. Horne, N. P. Meredith, S. A. Glauert, T. Moffat-Griffin, and J. C. Green (2010), Origin of energetic electron precipitation $>30 \mathrm{keV}$ into the atmosphere, J. Geophys. Res., 115, A00F08, doi:10.1029/ 2009JA014619. 
Mlynczak, M. G. (1997), Energetics of the mesosphere and lower thermosphere and the SABER experiment, Adv. Space Res., 20(6), 1177-1183, doi:10.1016/S0273-1177(97)00769-2.

Murtagh, D., et al. (2002), An overview of the Odin atmospheric mission, Can. J. Phys., 80, 309-319, doi:10.1139/p01-157.

Randall, C. E., et al. (2005), Stratospheric effects of energetic particle precipitation in 2003-2004, Geophys. Res. Lett., 32, L05802, doi:10.1029/ 2004GL022003.

Rodger, C. J., M. A. Clilverd, N. R. Thomson, R. J. Gamble, A. Seppälä, E. Turunen, N. P. Meredith, M. Parrot, J.-A. Sauvaud, and J.-J. Berthelier (2007), Radiation belt electron precipitation into the atmosphere: Recovery from a geomagnetic storm, J. Geophys. Res., 112, A11307, doi:10.1029/2007JA012383.

Rodger, C. J., M. A. Clilverd, J. C. Green, and M. M. Lam (2010), Use of POES SEM-2 observations to examine radiation belt dynamics and energetic electron precipitation into the atmosphere, J. Geophys. Res., 115, A04202, doi:10.1029/2008JA014023.

Rodgers, C. D. (2000), Inverse Methods for Atmospheric Sounding: Theory and Practice, World Sci., Singapore.

Rothman, L. S., et al. (2009), The HITRAN 2008 molecular spectroscopic database, J. Quant. Spectrosc. Radiat. Transfer, 110, 533-572, doi:10.1016/j.jqsrt.2009.02.013.

Rozanov, E., L. Callis, M. Schlesinger, F. Yang, N. Andronova, and V. Zubov (2005), Atmospheric response to $\mathrm{NO}_{\mathrm{y}}$ source due to energetic electron precipitation, Geophys. Res. Lett., 32, L14811, doi:10.1029/ 2005 GL023041.

Sandford, D. J., C. L. Beldon, R. E. Hibbins, and N. J. Mitchell (2010), Dynamics of the Antarctic and Arctic mesosphere and lower thermosphere-Part 1: Mean winds, Atmos. Chem. Phys., 10, 10,273-10,289, doi:10.5194/acp-10-10273-2010.

Seppälä, A., C. E. Randall, M. A. Clilverd, E. Rozanov, and C. J. Rodger (2009), Geomagnetic activity and polar surface air temperature variability, J. Geophys. Res., 114, A10312, doi:10.1029/2008JA014029.

Shimazaki, T. (1984), The photochemical time constants of minor constituents and their families in the middle atmosphere, J. Atmos. Terr. Phys., 46, 173-191, doi:10.1016/0021-9169(84)90143-0.
Siskind, D. E., G. E. Nedoluha, C. E. Randall, M. Fromm, and J. M. Russell III (2000), An assessment of southern hemisphere stratospheric $\mathrm{NO}_{\mathrm{x}}$ enhancements due to transport from the upper atmosphere, Geophys. Res. Lett., 27, 329-332, doi:10.1029/1999GL010940.

Solomon, S., P. J. Crutzen, and R. G. Roble (1982), Photochemical coupling between the thermosphere and the lower atmosphere: 1. Odd nitrogen from 50 to $120 \mathrm{~km}, J$. Geophys. Res., 87, 7206-7220, doi:10.1029/JC087iC09p07206.

Solomon, S. C., C. A. Barth, and S. M. Bailey (1999), Auroral production of nitric oxide measured by the SNOE satellite, Geophys. Res. Lett., 26, 1259-1262, doi:10.1029/1999GL900235.

Turunen, E., et al. (2009), Impact of different precipitation energies on $\mathrm{NO}_{\mathrm{x}}$ generation during geomagnetic storms, J. Atmos. Sol. Terr. Phys., 71, 1176-1189, doi:10.1016/j.jastp.2008.07.005.

Urban, J., et al. (2004), Moliere (v5): A versatile forward- and inversion model for the millimeter and sub-millimeter wavelength range, J. Quant. Spectrosc. Radiat. Transfer, 83(3-4), 529-554, doi:10.1016/S0022-4073(03) 00104-3.

Verronen, P. T., E. Turunen, T. Ulich, and E. Kyrölä (2002), Modelling the effects of the October 1989 solar proton event on mesospheric odd nitrogen using a detailed ion and neutral chemistry model, Ann. Geophys., 20, 1967-1976, doi:10.5194/angeo-20-1967-2002.

P. J. Espy, Department of Physics, Norwegian University of Science and Technology, N-7491 Trondheim, Norway.

P. Hartogh, Max Planck Institute for Solar System Research, Max-Planck-Str. 2, D-37191 Katlenburg-Lindau, Germany.

K. Holmén, Norwegian Polar Institute, Polar Environmental Centre, N-9296 Tromsø, Norway.

M. A. Clilverd, R. B. Horne, D. J. Maxfield, D. A. Newnham, and A. Seppälä, British Antarctic Survey, High Cross, Madingley Road, Cambridge CB3 0ET, UK. (david.newnham@bas.ac.uk)

C. J. Rodger, Department of Physics, University of Otago, PO Box 56, Dunedin, New Zealand. (crodger@physics.otago.ac.nz) 